\title{
Advanced Treatment and Imaging in Colonoscopy: The Pocket- Creation Method for Complete Resection and Linked Color Imaging for Better Detection of Early Neoplastic Lesions by Colonoscopy
}

\author{
Hironori Yamamoto ${ }^{1}$, Satoshi Shinozaki ${ }^{1,2}$, Yoshikazu Hayashi ${ }^{1}$, Yoshimasa Miura ${ }^{1}$, Tsevelnorov Khurelbaatar ${ }^{1}$, Hiroyuki Osawa ${ }^{1}$ \\ and Alan Kawarai Lefor ${ }^{3}$ \\ ${ }^{1}$ Division of Gastroenterology, Department of Medicine, Jichi Medical University, Shimotsuke, ${ }^{2}$ Shinozaki Medical Clinic, Utsunomiya, \\ ${ }^{3}$ Department of Surgery, Jichi Medical University, Shimotsuke, Japan
}

Early detection and resection of neoplastic lesions are key objectives to diminish colorectal cancer mortality. Resection of superficial colorectal neoplasms, cold snare polypectomy, endoscopic mucosal resection, and endoscopic submucosal dissection have all been developed and used worldwide. The pocket-creation method facilitates the resection of tumors in difficult and routine locations. Early detection is the most important first step to maximize the benefits of recent advancements in endoscopic techniques. However, the detection of small, flat-shaped, or faded color lesions remains difficult. Linked color imaging, a novel multi-light technology, facilitates the recognition of minor differences in tissue by enhancing the color contrast between early colorectal neoplasms and surrounding normal mucosa in a bright field of view. The most striking feature of linked color imaging is its ability to display the color of early neoplastic lesions as distinct from inflammatory changes, both of which have similar "redness" when viewed using white light imaging. To increase the detection rate of neoplasms, linked color imaging should be used from the outset for endoscopic observation. Early detection of superficial colorectal tumors can result in decreased mortality from colorectal cancer and maintain a good quality of life for patients. Clin Endosc 2019;52:107-113

Key Words: Endoscopy; Endoscopic submucosal dissection; Early detection; Cancer; Colorectal neoplasms

\section{INTRODUCTION}

Colorectal cancer is a major cause of cancer deaths throughout the world, with an increasing incidence. ${ }^{1}$ Despite significant advances in the treatment of colorectal cancer, the main strategy for the management of this disease is still early detection and resection. One of the major roles of colonoscopy is the management of colorectal cancer, including both di-

Received: October 23, 2018 Revised: November 23, 2018

Accepted: November 26, 2018

Correspondence: Hironori Yamamoto

Division of Gastroenterology, Department of Medicine, Jichi Medical University, 3311-1 Yakushiji, Shimotsuke, Tochigi 329-0498, Japan

Tel: +81-285-58-7347, Fax: +81-285-40-6598, E-mail: ireef@jichi.ac.jp ORCID: https://orcid.org/0000-0002-3601-1153

(cc) This is an Open Access article distributed under the terms of the Creative Commons Attribution Non-Commercial License (http://creativecommons.org/ licenses/by-nc/3.0) which permits unrestricted non-commercial use, distribution, and reproduction in any medium, provided the original work is properly cited. agnosis and treatment, and colorectal polypectomy decreases mortality. ${ }^{2}$

Advanced endoscopic resection techniques for the treatment of superficial neoplasms, such as snare polypectomy, endoscopic mucosal resection (EMR), and endoscopic submucosal dissection (ESD) are now available as minimally invasive curative treatments for many patients with early-stage colorectal cancer. Accurate pretreatment diagnosis using high-definition magnifying endoscopy with image-enhanced endoscopy, such as narrow-band imaging (NBI) and blue light imaging (BLI) is available for the selection of the most appropriate treatment strategy and determining the line of resection prior to endoscopic resection. However, these advancements in endoscopy can be applied only when neoplastic lesions are detected at an early stage. 


\section{ENDOSCOPIC RESECTION OF SUPERFICIAL COLORECTAL NEOPLASM}

Curative endoscopic resection of superficial tumors is limited to mucosal lesions and tumors with shallow submucosal invasion $(<1,000 \mu \mathrm{m})$. The quality of endoscopic resection is determined by careful evaluation of the specimen after resection. An en bloc resection with negative margins is always expected to achieve a curative resection. Although most local recurrences due to positive lateral margins can be managed by additional endoscopic resection during follow-up colonoscopy, a positive vertical margin spoils the clinical significance of endoscopic resection itself. To obtain a curative resection, an $\mathrm{R} 0$ resection (en bloc resection with negative margins), no lymphovascular invasion, budding grade 1 , and no deep submucosal invasion $(>1,000 \mu \mathrm{m})$ are required. ${ }^{3}$ Notably, the presence or absence of these factors is assessed with pathological evaluation of the submucosal layer to determine the necessity for additional surgical intervention. Therefore, the resected specimen ideally contains a thick submucosal layer. A large superficial tumor is usually treated with ESD. To achieve a high-quality ESD, an en bloc specimen with a thick submucosal layer is required.

\section{THE POCKET-CREATION METHOD}

To accomplish an efficacious and safe ESD throughout the alimentary tract, we developed the pocket-creation method (PCM; Fig. 1), which is routinely used for gastric, colorectal, and duodenal ESD in our institution. ${ }^{4-6}$ The standard approach is antegrade rather than retroflexed maneuvering. A key element of the PCM is a submucosal pocket creation with a minimal mucosal incision. Following the minimal mucosal incision, a circumferential incision is not made; however, a submucosal dissection is performed to create a pocket. The tip of the endoscope enters the pocket through the minimally incised mucosa. After entering the pocket, prudent dissection by identifying the deep submucosal layer is repeated to extend the pocket. The PCM facilitates selection of the exact plane of dissection and avoids dissecting at a superficial submucosal level that has plentiful blood vessels and adipose tissue. Dissection of the deep submucosal layer is a key element to obtain a good pathological specimen with a thick submucosal layer. During the procedure, additional injection of sodium hyaluronate just above the muscularis provides clear visualization of the muscularis through the endoscope hood. The volume of intraluminal gas is minimized to stabilize the maneuvering of the endoscope. During the last stage of the PCM, the pocket is opened after the complete dissection under the tumor. Consideration of the direction of gravity and a stepby-step mucosal incision with opening of the pocket from the inside to the luminal side should be kept in mind to avoid making the procedure difficult.

The PCM has four advantages as follows: (1) the injected solution is not dispersed because of a minimal incision; (2)
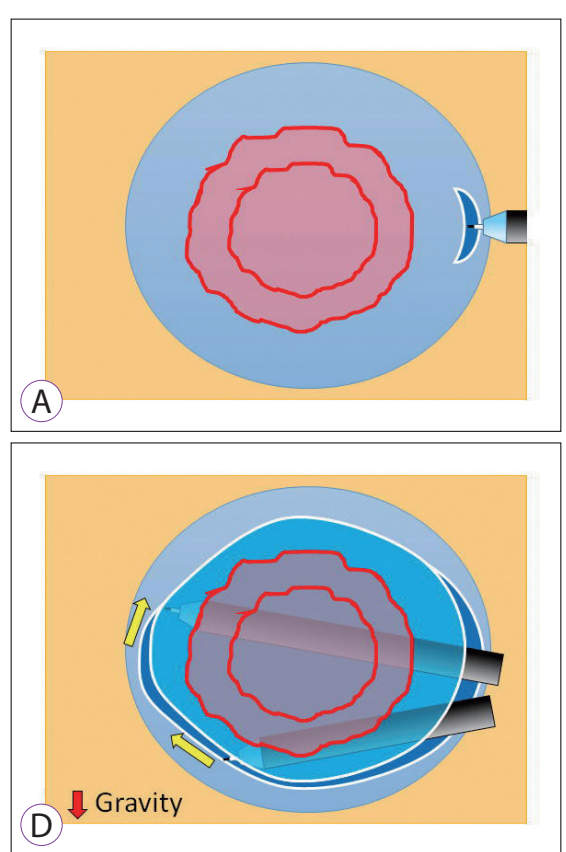
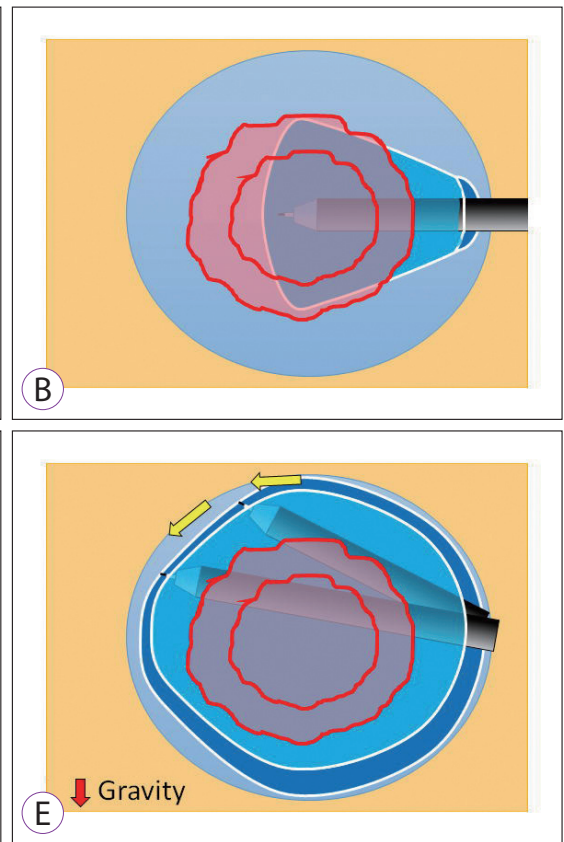
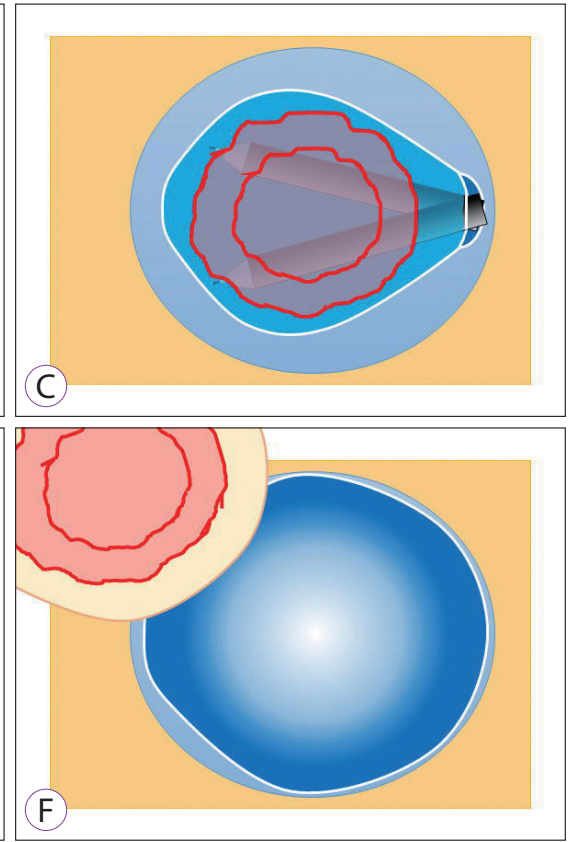

Fig. 1. Sequence for the pocket-creation method of endoscopic submucosal dissection. 
both traction and countertraction are simultaneously obtained because the transparent hood stretches the submucosal tissue in the limited space; (3) a vertical approach toward the muscularis can be changed to a horizontal approach; and (4) the influence of cardiopulmonary movement is less because of the synchronization between the endoscope and the pocket (Fig. 2). Unlike various techniques that require special devices such as clip-and-line, S-O clip, and external forceps to obtain traction, ${ }^{7-9}$ the PCM does not require any special devices except a transparent hood and electrosurgical knives. We use a small-caliber-tip transparent hood to obtain clear visualization in the pocket. ${ }^{10}$ Use of sodium hyaluronate is recommended to maintain a long-lasting submucosal cushion. ${ }^{6}$ The PCM can be performed as far as the endoscope reaches because it does not require special devices. Therefore, it can be applied for the ESD of duodenal lesions, which has been considered the most difficult ESD procedure. ${ }^{5}$ The PCM is not only useful for conquering difficulties such as duodenal ESD or a vertical approach but also for resecting routine lesions more easily using this universally applicable strategy.

\section{COLD SNARE POLYPECTOMY}

Cold snare polypectomy (CSP) has been widely disseminated to resect sub-centimeter colorectal polyps. We previously reported the efficacy and safety of CSP in comparison with conventional polypectomy, the so-called hot snare polypectomy (HSP). CSP significantly shortens the procedure time and shows a trend to decrease delayed bleeding as compared with HSP. ${ }^{11}$ CSP enables omission of HSP-specific procedures, such as placing a disposable electrode on the patient and performing electrocauterization at the time of polypectomy. Furthermore, CSP does not use cauterization that may extend areas of ulceration and injure submucosal arteries at the polypectomy site. $^{12,13}$

\section{EARLY DETECTION AND RESECTION}

By the early twenty-first century, minimally invasive resection methods, such as CSP, EMR, and ESD have been all widely used. ${ }^{12}$ The importance of early detection of gastrointestinal tract tumors has been emphasized over the last 20 years because minimally invasive endoscopic resection can only be used for premalignant or early-stage malignancies. Therefore, early detection is the most important first step to maximize the usefulness of recent advances in endoscopic resection techniques. However, the detection of small, flat-shaped, or faded-color lesions remains difficult. New image-enhanced endoscopic techniques facilitate the recognition of slight differences by enhancing the color contrast in a bright field of view.

\section{CHARACTERISTICS OF LINKED-COLOR IMAGING}

Linked color imaging (LCI) is a unique image-enhanced endoscopic technique that uses a novel multi-light technology developed by Fujifilm Company (Tokyo, Japan). LCI is available for both the laser endoscopic system (LASEREO) and the light-emitting diode endoscopic system (ELUXEO). LCI has the potential to increase the detection rate of adenomas and early colorectal cancers by enhancing the color differences between the neoplastic mucosa and the surrounding normal mucosa. As with previously developed technologies such as NBI and BLI, LCI enhances an image using pre-processing of narrow-band light irradiation followed by post-imaging signal processing. In the post-processing phase, acquired images are separated into blue, green, and red colors, which are then reallocated to enhance the color differentiation in an appropriate balance. Using this combination of colors, the red and white lesions become redder and whiter in LCI mode, respectively (Fig. 3). ${ }^{14}$

Compared with white light imaging (WLI), LCI enhances differences in the red color tone by advanced post-processing

\begin{tabular}{|c|c|c|c|}
\hline $\begin{array}{c}\text { Preventing dispersion } \\
\text { of solution }\end{array}$ & $\begin{array}{c}\text { Obtaining traction and } \\
\text { counter traction }\end{array}$ & $\begin{array}{c}\text { Conquering vertical and } \\
\text { downhill approach }\end{array}$ & $\begin{array}{c}\text { Synchronizing cardiac and } \\
\text { respiratory movement }\end{array}$ \\
\hline
\end{tabular}

Fig. 2. Advantages of the pocket-creation method (PCM). Minimal mucosal incision prevents dispersion of injected solution resulting in long-lasting maintenance of mucosal elevation. In the pocket, both traction and countertraction are obtained, and the tip of the endoscope is stabilized. Even in difficult locations, PCM enables stable submucosal dissection. A stabilized endoscope in the pocket synchronizes cardiac and respiratory movement. 
steps. The color correction limited to the red region emphasizes the contrast of the red color. The red color leads to an especially improved detection of inflammation with accurate delineation. We reported that LCI facilitates the detection of early gastric cancer by enhancing color contrast. ${ }^{15}$

However, the most striking feature of LCI is its ability to display the color of early neoplastic lesions as distinct from inflammatory changes that both have similar "redness" when imaged with WLI. Using LCI, the "redness" in areas of inflammatory change becomes purple, while neoplastic lesions remain red. This feature comes from the effect of 410-nm laser light in LASEREO and the violet light emitting diode in ELUXEO. The 410-nm or violet light can penetrate only a short distance from the mucosal surface and is specifically absorbed by hemoglobin. In neoplastic lesions, dilated microvasculature is concentrated in the superficial mucosa, and 410$\mathrm{nm}$ violet light is absorbed by hemoglobin in the concentrated vasculature (Fig. 3). Therefore, neoplastic areas of the mucosa look red on LCI imaging. In inflammatory mucosa, the dilated microvasculature is concentrated in the deep mucosa, and 410-nm violet light does not reach the vasculature and is reflected by the superficial layer without absorption (Fig. 3). Therefore, the mucosa in areas of inflammation looks violet on LCI imaging. Both neoplastic lesions and inflammatory mucosa have a similar level of "redness" when imaged with WLI. Consequently, LCI can distinguish neoplastic lesions from inflammatory mucosa. LCI provides good color contrast between neoplastic lesions and the surrounding mucosa by producing a bright and natural image, which is suitable for the detection of flat neoplastic lesions at a distant view.

\section{EARLY DETECTION OF COLORECTAL SUPERFICIAL LESION WITH LCI}

The miss rate for colorectal polyps is a great concern, even for well-trained colonoscopists. Heresbach et al. reported that a miss rate for colorectal polyps of $9 \%-28 \%$ was frequently associated with lesions in the left colon and sessile- or flatshaped polyps. ${ }^{16}$ Leufkens et al. also reported that a miss rate for colorectal polyps of $25 \%$ was significantly more frequently associated with adenomas in the right colon than in the left colon. ${ }^{17}$ Singh et al. reported that the mortality rate in patients with proximal colon cancer was not decreased with colonoscopy. ${ }^{18}$ Therefore, current routine colonoscopy may not be sufficiently sensitive to decrease the mortality of patients with right colon cancer, and colonoscopists should make every effort to increase the polyp detection rate in the right colon.

To improve the polyp detection rate in the right colon, some novel endoscopic equipment and add-on devices have been developed, including a transparent hood, balloon colonoscopy, wide-angle colonoscopy, and third-eye colonoscopy. Mat-

A

$\underline{\text { Pre-process }}$

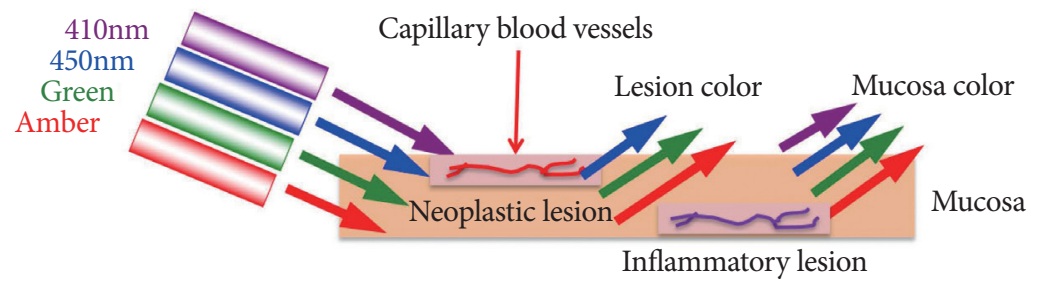

$\underline{\text { Post-process }}$

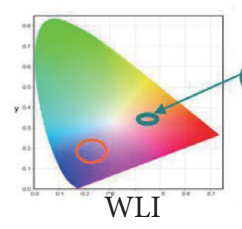

(B)

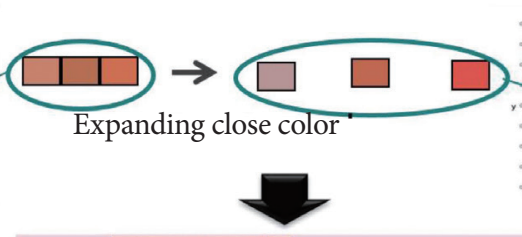

Lesion
New

illumination technologies

Fig. 3. (A) Pre-processing illumination technology of linked-color imaging (LCI): 410-nm violet light can penetrate only a short distance from the mucosal surface and is easily absorbed by hemoglobin. Typically, the dilated microvasculature is concentrated in the superficial mucosa in neoplastic lesions and in the deep mucosa in inflammatory lesions. Therefore, the 410-nm violet light is absorbed by neoplastic lesions but is reflected by inflammatory lesions. (B) The post-processing technology of LCl: color contrast is enhanced to make the red and white lesions become redder and whiter, respectively. WLI, white light imaging. 
sushita et al. reported that use of a transparent hood provided a $15 \%$ additional rate of polyp detection as compared with not using it. ${ }^{19}$ Use of a balloon colonoscope (G-EYE endoscope; Smart Medical Systems, Rảanana, Israel) had a significantly lower adenoma miss rate than conventional colonoscopy $(7.5 \%$ vs. $44.7 \%, p<0.001) .{ }^{20} \mathrm{~A}$ recent randomized trial reported that full-spectrum endoscopy had a significantly lower adenoma miss rate than standard forward-viewing colonoscopy (7\% vs. $41 \%, p<0.001) .{ }^{21}$ A prospective, multicenter, randomized-controlled trial reported that third-eye colonoscopy increased the adenoma detection rate by expanding the visual field behind the folds. ${ }^{22}$ Despite these greatly improved polyp detection rates, special endoscopic equipment or other add-on devices are required to use these techniques.

NBI and BLI are greatly useful to investigate known lesions by clearly visualizing their architecture and vascular pattern. However, these technologies may not be appropriate for screening endoscopy from a distant view because of insufficient light intensity to detect small or flat lesions in the stomach or colon, which have wide lumens. ${ }^{23}$ The color tones produced by NBI and BLI are quite different from WLI. Therefore, these narrow-band technologies are unlikely to be used from the beginning of observation, without WLI.

By contrast, the color tone produced by LCI is similar to white light, and LCI is as bright as or even brighter than WLI. Therefore, LCI can be used from the beginning of observation without needing to also use WLI. To increase the polyp detection rate, LCI should be used from the beginning before detecting suspicious lesions with other modalities. LCI is expected to have the potential to improve colorectal polyp detection rates, especially in the right colon and flat-shaped polyps. Fujimoto et al. analyzed the color differences among WLI, BLI, BLI-bright, and LCI using still endoscopic images containing a sessile serrated adenoma/polyp. ${ }^{24}$ Polyp detection and color differences were highest when using LCI, compared with the other image-enhanced technologies. ${ }^{24} \mathrm{~A}$ clearer visualization of the vascular network when using LCI allows endoscopists to avoid damaging submucosal vessels with resultant bleeding by selecting a less vascularized area during submucosal injection. ${ }^{25} \mathrm{~A}$ multicenter, randomized study from China reported a higher polyp detection rate with LCI colonoscopy than with WLI colonoscopy (91\% vs. $73 \%, p<0.001) .{ }^{26}$ We previously reported a patient with a laterally spreading tumor of the rectum that was clearly visualized as a bright red area on LCI, even at a distant view. ${ }^{14}$ A study from Japan using endoscopic images reported that the visibility score of colorectal non-granular flat tumors imaged with LCI was significantly higher than those imaged using WLI or BLI-bright. ${ }^{27}$ A study conducted using recorded video images reported that the polyp visibility scores when using LCI were significantly higher than those when using WLI for both expert and non-expert endoscopists. ${ }^{28}$ Screening colonoscopy with LCI improves the detection rate of small, flat-shaped, and/or faded-color colorectal lesions by enhancing the color contrast in a bright field of view.

Chromoendoscopy is a viable option to detect flat or small lesions using WLI at a distant view, but scattering indigo-carmine dye through the working channel is itself a time-consuming procedure. Unlike applying indigo-carmine dye, using the LCI mode requires just the push of a button. Taken together, BLI is effective for characterizing lesions with magnification, and LCI is good for early detection of small, flat, or faded-color neoplastic lesions. LCI can detect smaller colorectal polyps than WLI, which may increase the use of CSP and decrease the need for ESD or surgical resection (Figs. 4-8).

\section{CONCLUSIONS}

Endoscopic therapeutic procedures have progressed considerably. We can now select appropriate endoscopic resection techniques such as CSP, HSP, EMR, and ESD according to the size and nature of the neoplastic lesions. To make the most of these therapeutic techniques, endoscopic diagnosis has become more important than before. Of all the diagnostic procedures, detection is the most important first step.

LCI is a promising technology that has the potential to increase the adenoma detection rate during colonoscopy. LCI
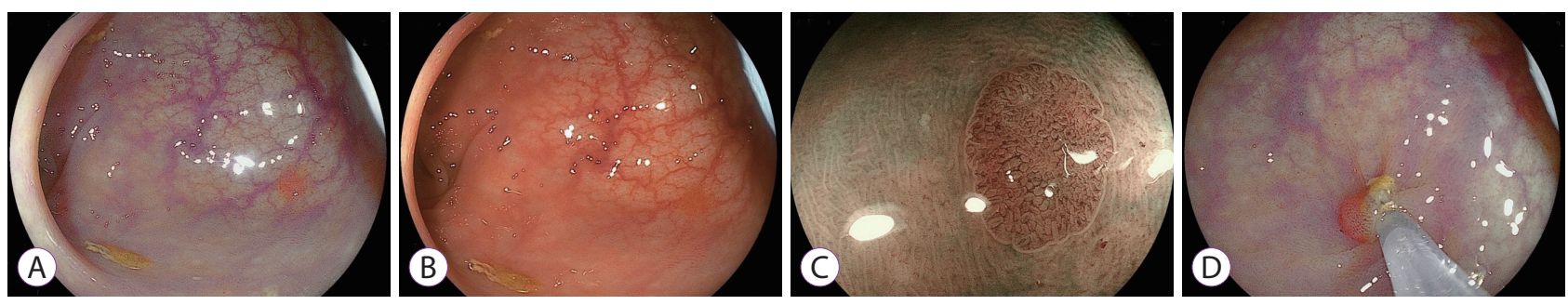

Fig. 4. A small sessile sigmoid colon polyp. (A) Easily detected by linked-color imaging. (B) Difficult detection by white-light imaging. (C) Blue laser imaging scan with magnification shows meshed capillaries compatible with a benign neoplastic lesion (JNET type 2A). (D) Cold-snare polypectomy. 
can enhance the color contrast of early colorectal neoplasms compared with the surrounding normal mucosa to improve the detection rate. To increase the detection rate, LCI should be used from the outset for endoscopic observation. Early detection leads to minimally invasive treatment such as CSP and ESD. The PCM facilitates colorectal ESD regardless of size, shape, and location, and consequently achieves a high success rate of complete en bloc resection. Early detection of superficial colorectal tumors and appropriate selection of endoscopic resection technique can result in decreasing colorectal cancer death rates and maintaining a good quality of life for patients.
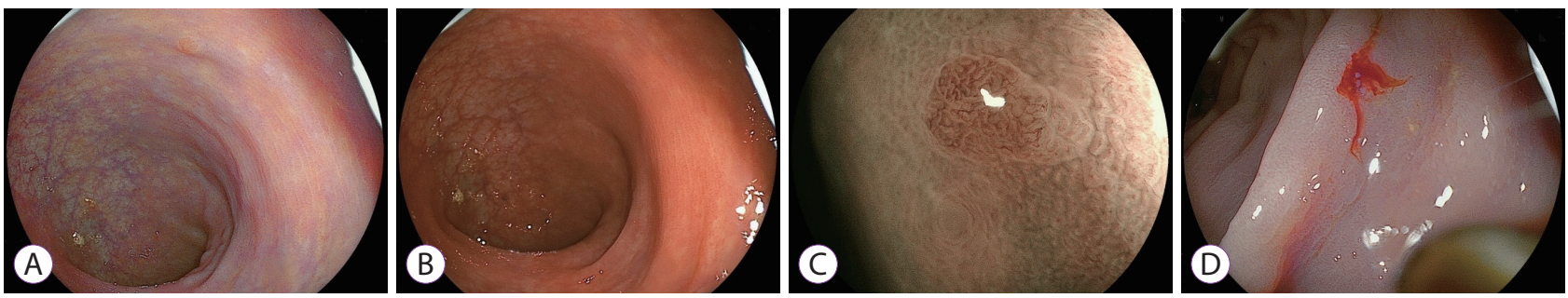

Fig. 5. A small sessile sigmoid colon polyp. (A) Easy detection by linked-color imaging. (B) Difficult detection by white-light imaging. (C) Blue laser imaging scan with magnification shows meshed capillaries (JNET type 2A). (D) Cold-snare polypectomy.
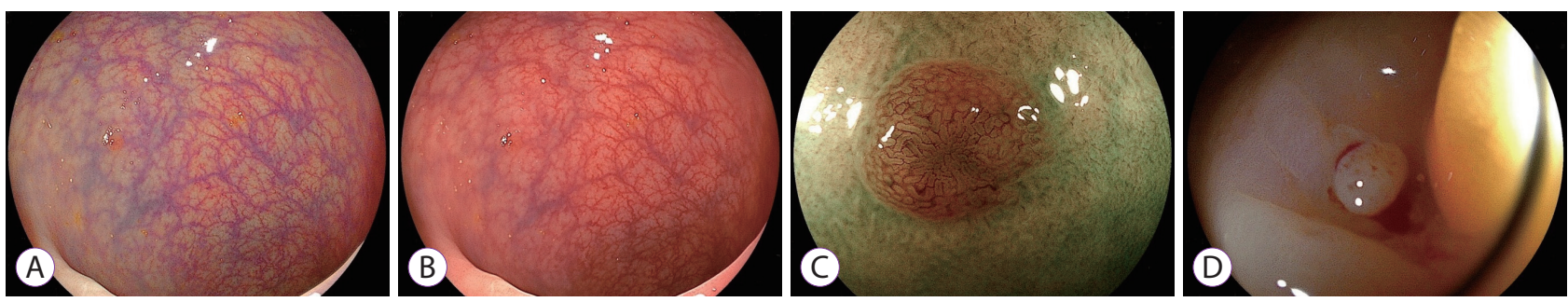

Fig. 6. A small sessile rectal polyp. (A) Easy detection by linked-color imaging. (B) Difficult detection by white-light imaging. (C) Blue laser imaging scan with magnification shows meshed capillaries (JNET type 2A). (D) Cold-snare polypectomy.
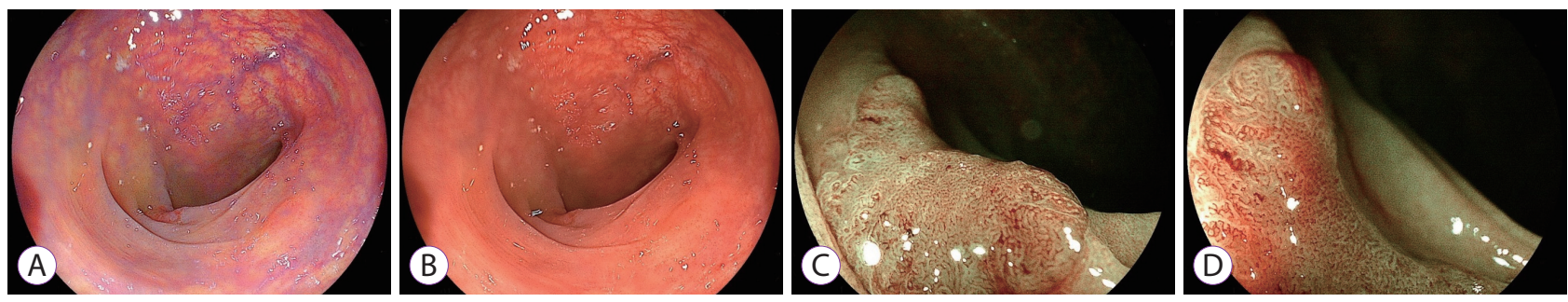

Fig. 7. Early colon cancer in the transverse colon. (A) Detectable using linked-color imaging. (B) Difficult detection by white light imaging. (C, D) Blue laser imaging scan with magnification shows irregular capillaries and an irregular surface pattern (JNET type 2B) that suggests superficial carcinoma.
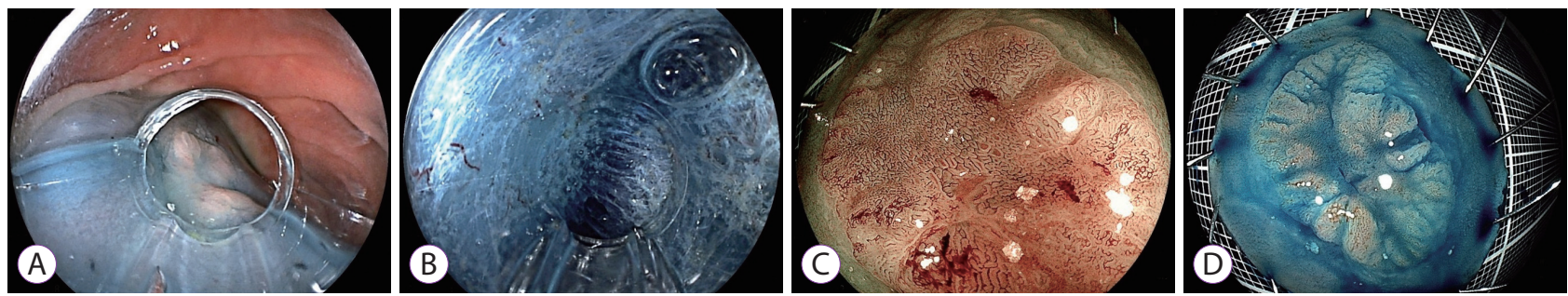

Fig. 8. Endoscopic submucosal dissection of a laterally spreading tumor of non-granular pseudo-depressed type (the lesion in Fig. 7), using the pocket-creation method. (A) Mucosal elevation using sodium hyaluronate. (B) Submucosal dissection in the pocket. (C) Blue laser imaging scan of the resected specimen shows irregular capillaries (JNET type 2B). (D) Chromoendoscopy image with indigocarmine dye spray of the resected specimen shows en bloc resection. Pathology: Well-differentiated tubular adenocarcinoma, $25 \times 21 \mathrm{~mm}$ in size, and intramucosal cancer with no lymphatic and venous invasions, and negative resection margins. A complete en bloc resection. 


\section{Conflicts of Interest}

Hironori Yamamoto has a consultant relationship with Fujifilm Corporation and has received honoraria, grants, and royalties from the company. Hiroyuki Osawa received honoraria from Fujifilm Corporation. Other authors have no financial conflicts of interest.

\section{REFERENCES}

1. Tanaka S, Kashida H, Saito Y, et al. JGES guidelines for colorectal endoscopic submucosal dissection/endoscopic mucosal resection. Dig Endosc 2015;27:417-434

2. Zauber AG, Winawer SJ, O’Brien MJ, et al. Colonoscopic polypectomy and long-term prevention of colorectal-cancer deaths. N Engl J Med 2012;366:687-696.

3. Watanabe T, Muro K, Ajioka Y, et al. Japanese Society for Cancer of the Colon and Rectum (JSCCR) guidelines 2016 for the treatment of colorectal cancer. Int J Clin Oncol 2018;23:1-34.

4. Sakamoto H, Hayashi Y, Miura Y, et al. Pocket-creation method facilitates endoscopic submucosal dissection of colorectal laterally spreading tumors, non-granular type. Endosc Int Open 2017;5:E123-E129.

5. Miura Y, Shinozaki S, Hayashi Y, Sakamoto H, Lefor AK, Yamamoto H. Duodenal endoscopic submucosal dissection is feasible using the pocket-creation method. Endoscopy 2017;49:8-14.

6. Miura Y, Hayashi Y, Lefor AK, Osawa H, Yamamoto H. The pocket-creation method of ESD for gastric neoplasms. Gastrointest Endosc 2016;83:457-458

7. Imaeda $\mathrm{H}$, Hosoe $\mathrm{N}$, Ida $\mathrm{Y}$, et al. Novel technique of endoscopic submucosal dissection by using external forceps for early rectal cancer (with videos). Gastrointest Endosc 2012;75:1253-1257.

8. Suzuki S, Gotoda T, Kobayashi Y, et al. Usefulness of a traction method using dental floss and a hemoclip for gastric endoscopic submucosal dissection: a propensity score matching analysis (with videos). Gastrointest Endosc 2016;83:337-346.

9. Ritsuno H, Sakamoto N, Osada T, et al. Prospective clinical trial of traction device-assisted endoscopic submucosal dissection of large superficial colorectal tumors using the S-O clip. Surg Endosc 2014;28:31433149.

10. Yamamoto H, Kawata H, Sunada K, et al. Successful en-bloc resection of large superficial tumors in the stomach and colon using sodium hyaluronate and small-caliber-tip transparent hood. Endoscopy 2003;35:690694.

11. Shinozaki S, Kobayashi Y, Hayashi Y, Sakamoto H, Lefor AK, Yamamoto H. Efficacy and safety of cold versus hot snare polypectomy for resecting small colorectal polyps: systematic review and meta-analysis. Dig Endosc 2018;30:592-599.

12. Shinozaki S, Hayashi Y, Lefor AK, Yamamoto H. What is the best therapeutic strategy for colonoscopy of colorectal neoplasia? Future perspectives from the East. Dig Endosc 2016;28:289-295.

13. Horiuchi A, Nakayama Y, Kajiyama M, Tanaka N, Sano K, Graham DY. Removal of small colorectal polyps in anticoagulated patients: a prospective randomized comparison of cold snare and conventional polypectomy. Gastrointest Endosc 2014;79:417-423.

14. Okada M, Sakamoto $H$, Takezawa $T$, et al. Laterally spreading tumor of the rectum delineated with linked color imaging technology. Clin Endosc 2016;49:207-208.

15. Fukuda H, Miura Y, Osawa $\mathrm{H}$, et al. Linked color imaging can enhance recognition of early gastric cancer by high color contrast to surrounding gastric intestinal metaplasia. J Gastroenterol 2018 Oct 5 [Epub]. https:// doi.org/10.1007/s00535-018-1515-6.

16. Heresbach D, Barrioz T, Lapalus MG, et al. Miss rate for colorectal neoplastic polyps: a prospective multicenter study of back-to-back video colonoscopies. Endoscopy 2008;40:284-290.

17. Leufkens AM, van Oijen MG, Vleggaar FP, Siersema PD. Factors influencing the miss rate of polyps in a back-to-back colonoscopy study. Endoscopy 2012;44:470-475.

18. Singh H, Nugent Z, Demers AA, Kliewer EV, Mahmud SM, Bernstein $\mathrm{CN}$. The reduction in colorectal cancer mortality after colonoscopy varies by site of the cancer. Gastroenterology 2010;139:1128-1137.

19. Matsushita M, Hajiro K, Okazaki K, Takakuwa H, Tominaga M. Efficacy of total colonoscopy with a transparent cap in comparison with colonoscopy without the cap. Endoscopy 1998;30:444-447.

20. Halpern Z, Gross SA, Gralnek IM, et al. Comparison of adenoma detection and miss rates between a novel balloon colonoscope and standard colonoscopy: a randomized tandem study. Endoscopy 2015;47:238-244.

21. Gralnek IM, Siersema PD, Halpern Z, et al. Standard forward-viewing colonoscopy versus full-spectrum endoscopy: an international, multicentre, randomised, tandem colonoscopy trial. Lancet Oncol 2014;15:353-360.

22. Leufkens AM, DeMarco DC, Rastogi A, et al. Effect of a retrograde-viewing device on adenoma detection rate during colonoscopy: the TERRACE study. Gastrointest Endosc 2011;73:480-489.

23. Kaminski MF, Hassan C, Bisschops R, et al. Advanced imaging for detection and differentiation of colorectal neoplasia: European Society of Gastrointestinal Endoscopy (ESGE) guideline. Endoscopy 2014;46:435449.

24. Fujimoto D, Muguruma N, Okamoto K, et al. Linked color imaging enhances endoscopic detection of sessile serrated adenoma/polyps. Endosc Int Open 2018;6:E322-E334.

25. Goda Y, Mori H, Kobara H, et al. Therapeutic application of linked color imaging for colorectal endoscopic mucosal resection. Endoscopy 2018;50:E8-E9.

26. Min M, Deng P, Zhang W, Sun X, Liu Y, Nong B. Comparison of linked color imaging and white-light colonoscopy for detection of colorectal polyps: a multicenter, randomized, crossover trial. Gastrointest Endosc 2017;86:724-730.

27. Suzuki T, Hara T, Kitagawa Y, et al. Linked-color imaging improves endoscopic visibility of colorectal nongranular flat lesions. Gastrointest Endosc 2017;86:692-697.

28. Yoshida N, Naito Y, Murakami T, et al. Linked color imaging improves the visibility of colorectal polyps: a video study. Endosc Int Open 2017;5:E518-E525. 